See discussions, stats, and author profiles for this publication at: https://www.researchgate.net/publication/233873616

\title{
Detection of a Biexciton in Semiconducting Carbon Nanotubes Using Nonlinear Optical Spectroscopy
}

Article in Physical Review Letters · November 2012

DOI: 10.1103/PhysRevLett.109.197402· Source: PubMed

\section{CITATIONS}

34

7 authors, including:

Leo Colombier

Ludwig-Maximilians-University of Munich

12 PUBLICATIONS 182 CITATIONS

SEE PROFILE

Emmanuel Rousseau

Université de Montpellier

51 PUBLICATIONS 1,171 CITATIONS

SEE PROFILE
Julien Selles

French National Centre for Scientific Research

17 PUBLICATIONS 134 CITATIONS

SEE PROFILE

Jean-Sébastien Lauret

Ecole normale supérieure de Paris Saclay

193 PUBLICATIONS 4,358 CITATIONS

SEE PROFILE

Some of the authors of this publication are also working on these related projects:

Optical properties of Carbon nanotubes View project

Photonics, plasmonics and meta-surfaces View project 


\title{
Biexciton in carbon nanotubes
}

\author{
L. Colombier, ${ }^{1,2}$ J. Selles, ${ }^{1,2}$ E. Rousseau, ${ }^{1,2}$ J. S. Lauret ${ }^{3}$ F. Vialla, ${ }^{4}$ C. Voisin, ${ }^{4}$ and G. Cassabois ${ }^{1,2, *}$ \\ ${ }^{1}$ Université Montpellier 2, Laboratoire Charles Coulomb UMR5221, F-34095, Montpellier, France \\ ${ }^{2}$ CNRS, Laboratoire Charles Coulomb UMR5221, F-34095, Montpellier, France \\ ${ }^{3}$ LPQM-ENS-Cachan, 61 avenue du Président Wilson, 94235 Cachan Cedex, France \\ ${ }^{4}$ Laboratoire Pierre Aigrain, Ecole Normale Supérieure, UPMC, Université Paris Diderot, \\ CNRS UMR8551, 24 rue Lhomond 75231 Paris Cedex 5, France
}

(Dated: July 17, 2012)

\begin{abstract}
We report the observation of the biexciton in semiconducting single-wall carbon nanotubes by means of nonlinear optical spectroscopy. Our measurements reveal the universal asymmetric lineshape of the Fano resonance intrinsic to the biexciton transition. For nanotubes of the $(9,7)$ chirality, we find a biexciton binding energy of $106 \mathrm{meV}$. From the calculation of the $\chi^{(3)}$ nonlinear response, we provide a quantitative interpretation of our measurements, leading to an estimation of the characteristic Fano factor $q$ of $7 \pm 3$. This value allows us to extract the first experimental information on the biexciton stability and we obtain a biexciton annihilation rate comparable to the exciton-exciton annihilation one.
\end{abstract}

PACS numbers: 78.67.Ch, 71.35.-y, 78.47.nd

Single-wall carbon nanotubes (SWNTs) are onedimensional nanostructures where the Coulomb interaction between the charge carriers strongly affects the opto-electronic properties. In semiconducting SWNTs the first elementary excitation consists in the creation of an electron-hole pair with a huge binding energy, reaching several tenths of $\mathrm{eV}[1-3]$. Compared to epitaxially grown semiconductor quantum wires [4], the binding of the so-called exciton is strongly enhanced in SWNTs, so that the optical response of SWNTs at room temperature is fully governed by the excitonic quasi-particles. As a matter of fact, their understanding is of great interest both from a fundamental point of view and for the development of carbon-based optoelectronic devices.

The recent observation of charged excitons in SWNTs [5] has brought the first experimental piece to an intensive debate initiated by theoretical studies of the binding of excitonic complexes and their stability in SWNTs [610]. The binding of one electron-hole pair and a hole (electron) in a positive (negative) trion, or the binding of two electrons and two holes in a biexciton have been predicted with energies as large as half to few hundreds of meVs, reminiscent of the non-perturbative exciton binding in SWNTs. However, the counterpart of the strong Coulomb correlations in SWNTs is the existence of efficient Auger processes that drive the population relaxation dynamics $[11,12]$. The evidence of trions by means of photoluminescence spectroscopy in chemically-[5] or optically-[13] doped SWNTs has indeed demonstrated that these processes do not hinder the observation of excitonic complexes. Moreover, it was suggested that localization effects may play an important role for keeping radiative yields high enough for detecting their photoluminescence signal [13]. On the contrary, there is so far no evidence for a biexcitonic signature in the optical response of SWNTs, thus raising fundamental issues on the difference between the excitonic complexes made of three (trion) or four (biexciton) charge carriers.
In this paper, we report the observation of the biexciton in semiconducting SWNTs by means of nonlinear optical spectroscopy. Our measurements reveal the universal asymmetric lineshape of the Fano resonance of the biexciton, with a biexciton binding energy of $106 \mathrm{meV}$ in SWNTs of the $(9,7)$ chirality. Our experiments consist in pump-probe spectroscopy under continuous-wave (cw) excitation in a SWNTs gelatin sample at 10K, where the biexciton manifests as an induced absorption resonance, higher in energy than the trion one. The identification of the biexciton is performed in a dual pump configuration where an additional excitation laser is directly tuned in resonance with the biexciton. The calculation of the $\chi^{(3)}$ nonlinear response allows us to perform a quantitative interpretation of our measurements and extract a characteristic Fano factor $q$ of $7 \pm 3$. This value brings the first experimental information on the Coulomb coupling efficiency between the biexciton and the continuum of free electron-hole pairs.

The exploration of the biexciton optical response is performed by means of optical nonlinear spectroscopy. In Fig.1(a), we display the energy level diagram of the exciton and biexciton states, and the pump-probe scheme implemented for our measurements. Under resonant excitation of the fundamental $\mathrm{S}_{11}$ excitonic state, labelled $|X\rangle$, the pump laser of energy $E_{P}$ opens an absorption channel for the probe laser at an energy detuning $E_{\text {probe }}-E_{P}$ given by the binding energy of the biexciton state labelled $|X X\rangle$. As pointed out in the pioneering work of Maruani and Chemla [14], the biexciton level diagram is a model system in semiconductor physics for the observation of a Fano resonance. This effect arises from the quantum interference between two transitions that are further coupled by a specific interaction. In the case of the biexciton, the absorption of one photon from the exciton state $|X\rangle$ leads either to the formation of a biexciton $|X X\rangle$, or to the excitation of the bound electron-hole pair to a state 


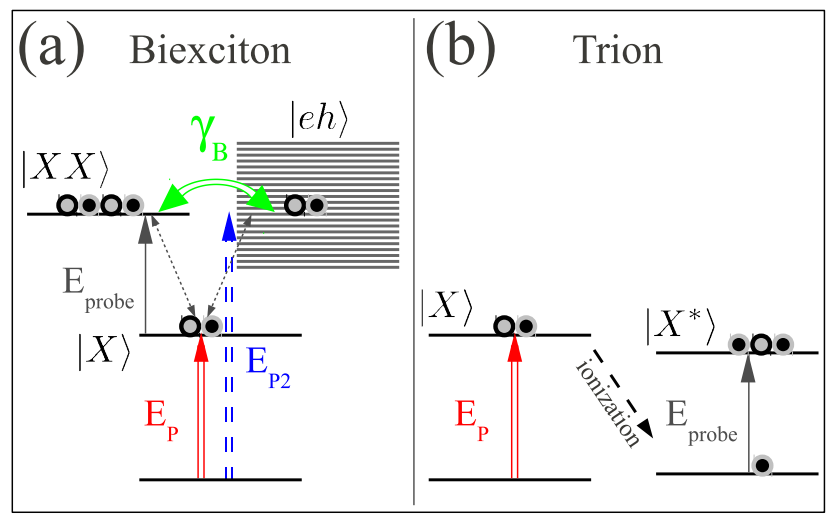

FIG. 1. Energy level diagram displaying the biexciton (a) and trion (b) transitions probed in our nonlinear optical experiments. $E_{P}$ and $E_{P 2}$ stand for the energy of the first and second pump lasers, respectively, $E_{\text {probe }}$ for the energy of the probe laser, and $\gamma_{B}$ is the biexciton annihilation rate characterizing the Coulomb coupling efficiency between the biexciton state $|X X\rangle$ and the $\mathrm{S}_{11}$ continuum of free electron-hole pair states $|e h\rangle$.

$|e h\rangle$ belonging to the $\mathrm{S}_{11}$ continuum of free electron-hole pairs (Fig.1(a)). The coupling between the $|X X\rangle$ and $|e h\rangle$ states is the biexciton annihilation process. A Fano resonance leads to an asymmetric lineshape, as observed in our measurements and quantitatively analyzed at the end of this paper. Before the presentation of our experiments, we further comment on the existence of a nonlinear signal for the trion state, labelled $X^{*}$, as recently reported in Ref.[13]. In Fig.1(b), we see that under resonant excitation of the exciton state $|X\rangle$, the pump laser may also open an absorption channel for the probe laser, at an energy detuning $E_{\text {probe }}-E_{P}$ given by the trion binding energy, if the electron-hole pair of the exciton has dissociated and if one charge carrier has left the nanotube. The existence of an ionization process is thus a strict requirement to the observation of the trion transition in optical measurements performed in nominally undoped SWNTs [13]. The experimental data presented in the latter study further suggest the localization of the trion in order to prevent its migration to photo-induced quenching sites, detrimental to the radiative properties of this excitonic complex. In summary, while both excitonic complexes give rise to induced absorption, which manifests as a negative signal in differential transmission experiments, we stress that in the case of the trion, the nonlinear signal is of extrinsic nature whereas it is intrinsic for the biexciton.

Our sample consists of isolated SWNTs synthetized by laser ablation of a doped graphite target, and embedded in a gelatin, following the procedure described in Ref.[15]. The optical characterization displayed in Fig.2 is performed by photoluminescence spectroscopy of our carbon nanotubes gelatin sample at 10K. The excitation is provided by a cw Ti:sapphire laser and the detector consists of an InGaAs photodiode-array. Following the approach introduced by Bachilo et al., we assign each peak in the two-dimensional map of the photoluminescence signal intensity to a given chiral species [16]. In the spectral domain explored in our nonlinear measurements, the optical response is dominated by the $(12,5)$ and $(9,7)$ chiralities. While the $\mathrm{S}_{22}$ excitonic transition has approximately the same energy of $1.53 \mathrm{eV}$ for both chiralities (Fig.2), the $\mathrm{S}_{11}$ excitonic transition is centered at 0.85 and $0.95 \mathrm{eV}$ for the $(12,5)$ and $(9,7)$ SWNTs, respectively. The inhomogeneous linewidth in emission is about $45 \mathrm{meV}$.

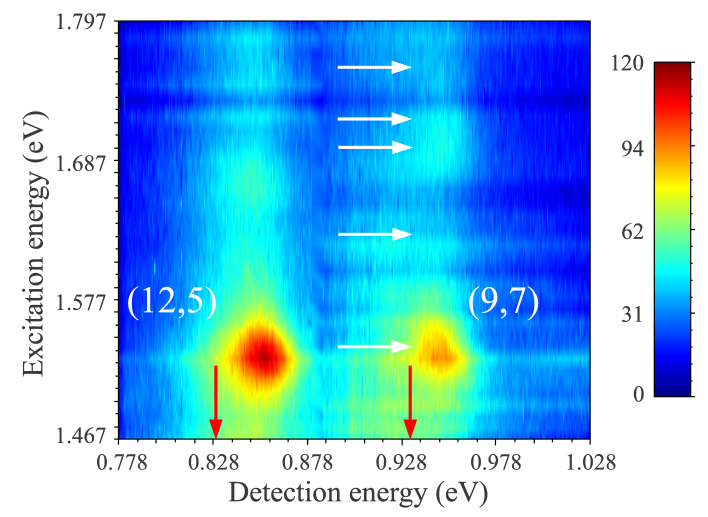

FIG. 2. Two-dimensional map of the photoluminescence signal intensity in the carbon nanotubes gelatin sample at $10 \mathrm{~K}$. The $(12,5)$ and $(9,7)$ chiralities dominate the optical response in the spectral domain investigated below by nonlinear optical spectroscopy. The vertical (red) arrows indicate the energy $E_{P}$ of the first pump used in Fig.3. The horizontal (white) arrows indicate the energy $E_{P 2}$ of the additional second pump used in Fig.4 (see energy level diagram in Fig.1).

Our optical nonlinear measurements are based on differential transmission experiments in a spectrally resolved cw pump-probe configuration, adapted from the spectral-hole burning setup previously used for the study of exciton-exciton [17] and exciton-phonon [18] interactions in SWNTs. Our original nonlinear technique relies on a $\mathrm{cw}$ laser excitation, that leads to the selective excitation of SWNTs of a given chirality in ensemble measurements. The probe laser is an external-cavity semiconductor laser of energy $E_{\text {probe }}$ tunable from $0.74 \mathrm{eV}$ to $0.84 \mathrm{eV}$, with a spectral width below $100 \mu \mathrm{eV}$. The pump laser is a semiconductor distributed feed-back laser of energy $E_{P}$, where $E_{P}$ is either $0.83 \mathrm{eV}$ or $0.93 \mathrm{eV}$. The use of these two excitation energies allows the exploration of energy detuning $E_{\text {probe }}-E_{P}$ ranging from $-190 \mathrm{meV}$ to $+10 \mathrm{meV}$. Note that for $E_{P}=0.83 \mathrm{eV}$ (left vertical red arrow in Fig.2), the pump laser is resonant with $(12,5)$ SWNTs, while for $E_{P}=0.93 \mathrm{eV}$ (right vertical red arrow 
in Fig.2), the pump laser excites SWNTs of the $(9,7)$ chirality.

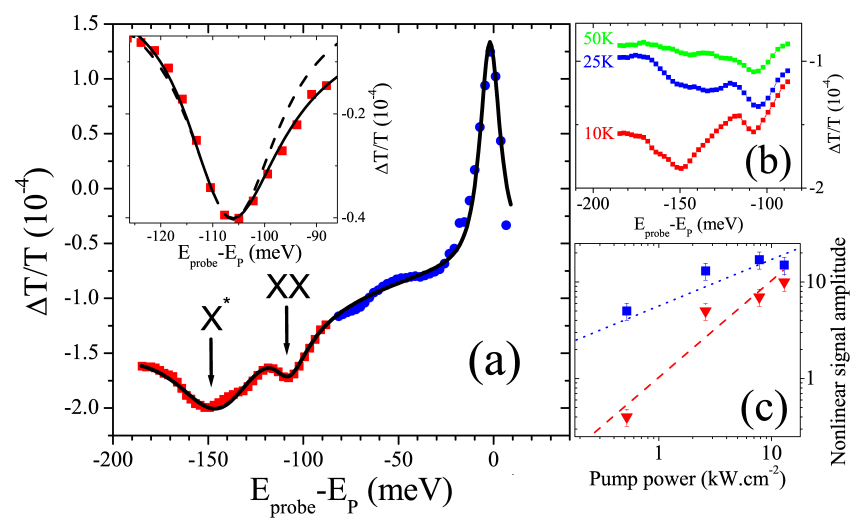

FIG. 3. (a) Differential transmission $\Delta T / T$ of the probe laser at $10 \mathrm{~K}$, as a function of the energy detuning $E_{\text {probe }}-E_{P}$ between the probe and first pump lasers. $E_{P}=0.83 \mathrm{eV}$ for the right part (blue dots), and $E_{P}=0.93 \mathrm{eV}$ for the left one (red squares) (see Fig.2). The solid (black) line is a fit of the experimental data. Inset: expanded view of the biexciton line after subtracting the linear background and the induced absorption signal of the trion (described in the text). The dashed line is a fit of the experimental data in the Lorentzian limit $(q=+\infty)$. (b) Temperature dependence of the differential transmission $\Delta T / T$ for $E_{P}=0.93 \mathrm{eV}$, between $10 \mathrm{~K}$ and $50 \mathrm{~K}$ (the spectrum at $25 \mathrm{~K}$ is shifted by +0.2 for clarity). (c) Pump power dependence of the nonlinear signal amplitude for the $X X$ line (blue squares), and $X^{*}$ line (red triangles).

In Fig.3(a), we present our differential transmission measurements at $10 \mathrm{~K}$, for which the excitation density of the pump is $15 \mathrm{~kW} . \mathrm{cm}^{-2}$. When $E_{\text {probe }}-E_{P} \sim 0$, our measurements are performed in a degenerate configuration where nonlinear spectral-hole burning occurs within the SWNTs ensemble [17]. As discussed in details in Ref.[17], the differential transmission signal $\Delta T / T$ exhibits positive values, corresponding to the photobleaching of the fundamental $\mathrm{S}_{11}$ excitonic transition. This component has an amplitude of $2 \times 10^{-4}$ and it is superimposed on a negative signal, which corresponds to a residual nonresonant photo-absorption which magnitude smoothly increases for larger negative detuning. The footprints of excitonic complexes appear when exploring energy detuning $E_{\text {probe }}-E_{P}$ ranging from $-90 \mathrm{meV}$ to $-190 \mathrm{meV}$. We observe two negative lines, with an amplitude of the order of few $10^{-5}$, that correspond to pumpinduced absorption, as expected for the biexciton and trion states (Fig.1). The resonances appear at energy detunings $E_{\text {probe }}-E_{P}$ of $-150 \mathrm{meV}$ and $-106 \mathrm{meV}$, and we show below how they can be attributed the trion $X^{*}$ and the biexciton $X X$, respectively.

The first point deals with the values of the binding energies. In $(9,7)$ SWNTs, Matsunaga et al. and Santos et al. reported a trion binding energy of $135 \mathrm{meV}$ [5] and $130 \mathrm{meV}$ [13], respectively. In our case, we measure a slightly larger value of $150 \mathrm{meV}$. This $15-20 \mathrm{meV}$ redshift of the trion line is similar to the one reported in Ref. [15] for the exciton line when comparing SWNTs in an aqueous suspension and in a gelatin matrix. Assuming that the trion binding energy follows the same scaling law in $\epsilon^{-1.4}$ as the exciton one [1] (where $\epsilon$ is the effective dielectric constant of the nanotube environment), this corresponds to a relative change of the dielectric constant of the order of $10 \%$, as in Ref. [15]. Besides, recent theoretical calculations have predicted a smaller binding energy for the biexciton than for the trion, with a ratio ranging from 0.7 to 0.85 [10]. From our experimental data, we obtain a value of 0.71 , in agreement with the predictions of Ref.[10]. Experimental measurements as a function of temperature further support our interpretation. The induced absorption line at $-106 \mathrm{meV}$ displays the same temperature dependence in terms of broadening and amplitude as the excitonic photo-bleaching line [21], which was the focus of Ref.[18]. The strong similarity between the biexciton and the exciton is also observed in semiconductor quantum dots, and it indicates the correlation of the thermally-assisted broadening processes involved in the genuine biexciton-exciton cascade [19]. On the other hand, the amplitude of the nonlinear signal decreases more rapidly with temperature for the trion than for the biexciton, as shown in Fig.3(b). Following Ref.[13] concluding to the presence of specific localization effects for the detection of the trion emission signal, we interpret this phenomenology as due to a thermally-assisted carrier escape, resulting in a partial neutralization of the nanotube which reduces the pump-induced absorption signal at the energy of the trion. Finally, as far as the pumppower dependence is concerned, we observe in Fig.3(c) that the magnitude of the XX line displays the same sublinear increase as for the photo-bleaching of the excitonic transition [17]. This is what is expected for the biexciton induced absorption which scales like the density of photo-generated excitons [20]. On the other hand, the trion nonlinear signal exhibits again a different behavior with a merely linear dependence (Fig.3(c)) similar to the variations of the trion photoluminescence signal in the all-optical generation measurements of Ref.[13]. All these facts strongly support the assignment of the lines in Fig.3(a) to the trion and biexciton complexes.

In order to further confirm the identification of the pump-induced absorption line at $-106 \mathrm{meV}$ as due to the biexciton, we have experimentally tested a fundamental difference between the trion and biexciton states in terms of energy. The trion is an elementary excitation of a carbon nanotube with a residual charge carrier, and its energy is given by the exciton energy lowered by the trion binding energy. The biexciton is an elementary excitation of a neutral nanotube, and its energy is on the contrary twice the exciton energy lowered by the biexciton binding energy (Fig.1). In other words, in the context 


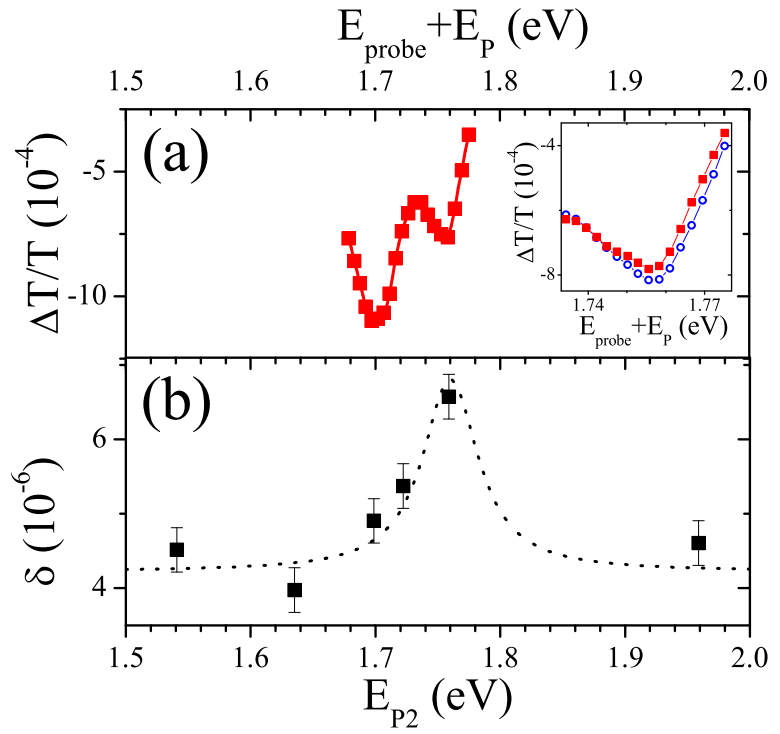

FIG. 4. (a) Differential transmission $\Delta T / T$ of the probe laser at $10 \mathrm{~K}$, as a function of the sum of the probe energy $E_{\text {probe }}$ and $E_{P}=0.93 \mathrm{eV}$. (Inset) Differential transmission $\Delta T / T$ spectrum in the biexciton spectral range, with (open blue circles) and without (full red squares) the additional pump laser at an energy $E_{P 2}=1.76 \mathrm{eV}$. (b) Deformation $\delta$ of the differential transmission $\Delta T / T$ spectrum as a function of the energy $E_{P 2}$ of the additional pump laser. The dashed line is a guide for the eyes of Lorentzian form.

of our nonlinear experiments, the energy of the biexciton quasi-particle is given by the sum $E_{\text {probe }}+E_{P}$, which is about $1.76 \mathrm{eV}$, while for the trion, the quasi-particle energy is simply given by $E_{\text {probe }}$, which is of the order of $0.78 \mathrm{eV}$. On the basis of this marked difference, we have tried to perform a resonant excitation of the biexciton around the energy of $1.76 \mathrm{eV}$. More precisely, we have carried out additional nonlinear measurements in an original dual-pump configuration where a second pump laser is used to resonantly excite the biexciton at an energy $E_{P 2}$ spanning the sum $E_{\text {probe }}+E_{P}$ of the probe and first pump laser energies (Fig.1(a)). The second pump laser consists either of a $\mathrm{cw}$ Ti:sapphire laser with $E_{P 2}$ ranging from $1.47 \mathrm{eV}$ to $1.76 \mathrm{eV}$, or of a HeNe laser of energy $E_{P 2}$ equal to $1.96 \mathrm{eV}$. We have studied the differential transmission $\Delta T / T$ spectrum with (open blue circles, inset of Fig.4(a)) and without (full red squares, inset of Fig.4(a)) the additional pump laser, as a function of its energy $E_{P 2}$, for a constant excitation density of $1.5 \mathrm{~kW} . \mathrm{cm}^{-2}$. As shown in the inset of Fig.4, there is a sizeable modification of the nonlinear signal intensity. In order to carefully characterize the variations of the differential transmission $\Delta T / T$ spectrum with $E_{P 2}$ we have evaluated $\delta$, defined as the absolute value of the deformation of the nonlinear spectrum in the biexciton spectral range [21]. As seen in Fig.4(b), $\delta$ displays a systematic variation as a function of $E_{P 2}$ with a maximum recorded value at the energy of the biexciton, and no other resonance that would correspond to the $X^{*}$ line or to the $\mathrm{S}_{22}$ excitonic state centered at $1.53 \mathrm{eV}$ (Fig.2). This latter observation rules out any trivial effect related to the density of additional excitons photogenerated by the second pump laser. In fact, the variation of $\delta$ with $E_{P 2}$ is not correlated to the excitation spectrum of the photoluminescence signal of the $(9,7)$ SWNTs, as seen from the horizontal (white) arrows in Fig.2 indicating the energies $E_{P 2}$ of the second pump laser. On the contrary, the deformation $\delta$ is found to be maximum precisely when $E_{P 2}$ matches the expected value of $1.76 \mathrm{eV}$ of the biexciton (Fig.4(a)), thus confirming the identification of the biexciton in our nonlinear experiments. As far as the physical processes involved in our dual-pump nonlinear experiments are concerned, several interpretations are under consideration. In a perfect nanotube, the direct one-photon excitation of the biexciton is supposed to be forbidden [9]. However, any breakdown of this selection rule due to the nanotube deformation or the presence of defects can lead to a direct photogeneration of biexcitons, competing with the pump-induced absorption process. A more straightforward way of modifying the biexciton spectrum is the direct dipole-allowed photogeneration of free electron-hole pairs at the energy of the biexciton. This mechanism is expected to perturb the Coulomb coupling between $|X X\rangle$ and $|e h\rangle$ responsible for the biexciton annihilation. This interaction is at the heart of the Fano resonance profile of the biexciton, which is the focus of the last part of the paper. The identification of the biexciton being confirmed by our dual pump nonlinear experiments, we eventually proceed with a quantitative analysis of the biexciton asymmetric lineshape.

As demonstrated by Maruani and Chemla in bulk $\mathrm{CuCl}$ and $\mathrm{CdS}$ semiconductors [14], biexciton in semiconductor materials provide a text-book example of a Fano resonance. The quantum interference between the two competing pathways (Fig.1(a)) is highly sensitive to the Fano factor $q$ which characterizes the interaction between the final states and which controls the Fano resonance asymmetry. In the context of biexciton in SWNTs, the Fano factor $q$ is determined by the Coulomb coupling (denoted $\gamma_{B}$ in Fig.1(a)) between $|X X\rangle$ and $|e h\rangle$ responsible for the biexciton annihilation. In order to evaluate the Fano factor $q$, we have performed the theoretical calculations of the nonlinear signal detected in our pump-probe measurements. In the framework of the $\chi^{(3)}$ nonlinear response developed in Ref.[14], we have computed the theoretical differential transmission $\Delta T / T$ spectrum in the case of an inhomogeneously broadened absorption line [21]. A phenomenological negative background linearly increasing with detuning and a negative Lorentzian line for the trion were added to our $\chi^{(3)}$ calculations, which intrinsically account for the excitonic photo-bleaching around zero detuning and for the biexcitonic pump-induced absorption around -106 meV. With 
this procedure, we reach a good agreement with our experimental data (Fig.3(a)). In particular, we fairly reproduce the asymmetric lineshape of the biexciton (Fig.3(a), inset) from which we extract a Fano factor $q$ of $7 \pm 3$. The consideration of negative values of $q$ leads to an asymmetric profile with a low-energy tail, which is inconsistent with our measurements systematically showing a highenergy tail, i.e. positive values of $q$ (Fig.3(a), inset). As far as higher values of $q$, the limit $q=+\infty$ consists in a vanishing coupling to the continuum, resulting to a simple Lorentzian model without any Fano effect. This limit corresponds to the dashed line in the inset of Fig.3(a), which represents the best fit of our experimental data when varying the slope of the background and the width of the trion line. Actually, finite positive values of $q$ are mandatory in order to account for the asymmetry of the biexciton spectral profile (Fig.3(a), inset). Finally, we obtain an excitonic homogeneous linewidth $\Gamma_{X}=11 \pm 2 \mathrm{meV}$ and a comparable one for the biexciton $\Gamma_{X X}=11 \pm 2 \mathrm{meV}$, thus ruling out any significant difference in the coherence relaxation dynamics of the exciton and biexciton states.

The situation is assumed to be, on the contrary, radically different for the population relaxation dynamics since the radiative yield of the biexciton has been so far too low for the detection of any photoluminescence signal. The lack of evidence for a biexciton photoluminescence signal raises fundamental questions on the biexciton stability $[6,7,9,13]$ in the context of the strong efficiency of Auger processes in SWNTs [11, 12]. In fact, our evaluation of the Fano factor provides a unique way for an estimation of the biexciton annihilation rate $\gamma_{B}$. According to the $1 / \mathrm{L}$ scaling law in one-dimensional systems, we rewrite $\gamma_{B}$ as $\frac{B}{L}$, while the rate of the exciton-exciton annihilation process is written as $\gamma_{A}=\frac{A}{L}$ [11]. From the expression of the Fano factor, $q=\frac{\mu_{X X}}{\mu_{e h}} \frac{1}{\pi \rho \hbar \gamma_{B}}$, where $\mu_{X X}$ and $\mu_{e h}$ are the dipole moments of the two competing pathways (Fig.1) and $\rho$ is the joint density of $|e h\rangle$ states, we deduce $B \sim 1 \mathrm{ps}^{-1} . \mu \mathrm{m}$ [21]. In a first approximation, this value is identical to the typical $A$ one for excitonexciton annihilation, ranging from 0.24 to $2 \mathrm{ps}^{-1}$. $\mu \mathrm{m}[22-$ 24]. As a matter of fact, our analysis surprisingly reveals that the Auger processes responsible for the biexciton annihilation have an efficiency comparable to the excitonexciton annihilation one. In our SWNTs with a length of the order of $500 \mathrm{~nm}$, we obtain a biexciton annihilation time of about $0.5 \mathrm{ps}$, to be compared to the ps-time scale of the exciton-exciton annihilation [11, 12]. However, although Auger recombinations of exciton and biexciton occur on similar temporal scales, the former results in a remaining exciton with a lifetime in the nanosecond range while the latter leads to the irreversible decay of the biexciton state. This gives a new insight for understanding the current absence of a photoluminescence signal from the biexciton in the prospect of its future detection in emission.

In conclusion, we have reported the observation of the biexciton in semiconducting single-wall carbon nanotubes. Our measurements reveal the universal asymmetric lineshape of the Fano resonance intrinsic to the biexciton transition. For nanotubes of the $(9,7)$ chirality, we find a biexciton binding energy of $106 \mathrm{meV}$. The calculation of the $\chi^{(3)}$ nonlinear response allows us to perform a quantitative interpretation of our measurements and extract a characteristic Fano factor of $7 \pm 3$. We find that the biexciton annihilation process has the same efficiency as the exciton-exciton annihilation one, thus raising fundamental questions for the quest of a biexciton photoluminescence signal and the evidence for a biexciton-exciton quantum cascade.

We gratefully acknowledge S. Rousset, P. Valvin, C. L'Henoret for technical support, T. Guillet and B. Gil for helpful discussions. This work was financially supported by the Conseil Scientifique de l'Université Montpellier 2.

*e-mail: Guillaume.Cassabois@univ-montp2.fr
[1] V. Perebeinos et al., Phys. Rev. Lett. 92, 257402 (2004).

[2] F. Wang et al., Science 308, 838 (2005).

[3] J. Maultzsch et al., Phys. Rev. B 72, 241402(R) (2005).

[4] M. Bayer, S. N. Walck, T. L. Reinecke, and A. Forchel, Phys. Rev. B 57, 6584 (1998).

[5] R. Matsunaga et al., Phys. Rev. Lett. 106, 037404 (2011).

[6] T.G. Pedersen et al., Nano Lett. 5, 291 (2005).

[7] D. Kammerlander et al., Phys. Rev. Lett. 99, 126806 (2007).

[8] I.V. Bondarev, Phys. Rev. B 83, 153409 (2011).

[9] K. Watanabe and K. Asano, Phys. Rev. B 83, 115406 (2011).

[10] K. Watanabe and K. Asano, Phys. Rev. B 85, 035416 (2012).

[11] F. Wang et al., Phys. Rev. B 70, 241403(R) (2004).
[12] Y-Z. Ma et al., Phys. Rev. Lett. 94, 157402 (2005).

[13] S. Santos et al., Phys. Rev. Lett. 107, 187401 (2011).

[14] A. Maruani and D.S. Chemla, Phys. Rev. B 23, 841 (1981).

[15] S. Berger et al., J. Appl. Phys. 105, 094323 (2009).

[16] S.M. Bachilo et al., Science 298, 2361 (2002).

[17] D.T. Nguyen et al., Phys. Rev. Lett. 107, 127401 (2011).

[18] D.T. Nguyen et al., Phys. Rev. B 84, 115463 (2011).

[19] F. Gindele et al., Phys. Rev. B 60, 2157 (1999).

[20] A.S. Lenihan et al., Phys. Rev. B 69, 045306 (2004).

[21] see the Supplemental Material for more details.

[22] F. Wang et al., Phys. Rev. B 73, 245424 (2006).

[23] L. Valkunas et al., Phys. Rev. B 73, 115432 (2006).

[24] Y-F. Xiao et al., Phys. Rev. Lett. 104, 017401 (2010). 